\title{
A Context-Aware Location Service for Metropolitan Ad Hoc Networks
}

\author{
Giovanni Turi \\ IIT Institute, CNR, \\ Via G. Moruzzi 1, 56124 Pisa, Italy \\ Giovanni.Turi@iit.cnr.it
}

\begin{abstract}
This paper presents a node location service for metropolitan Ad Hoc networks. The new system is innovative because it exploits context information in its internal polices. Context information is referred as knowledge about metropolitan user mobility patterns. In urban areas people do not uniformly distribute. Specific zones, defined as hot spots, work like assembly points, where people show predictive mobility. This zones are office buildings, shopping malls and similar operational contexts. The paper shows how a hot spots aware node location service could better find nodes where to publish and retrieve updated position. This proposal paper mainly focuses on protocol aspects, showing mechanisms to let the system adaptable to the inherent dynamic conditions of an Ad Hoc network.
\end{abstract}

\section{Introduction}

Infrastructureless mobile networks, also called Ad Hoc networks, are systems where mobile and resource-constrained nodes organize spontaneously, forming a network with dynamic topology. With an increasing use, in urban areas, of ubiquitous technologies, like PDA's, smart phones and wearable computers, users will hopefully form Ad Hoc networks on a metropolitan scale. In this scenario, tracking and delivering the current node positions is an important task that a node location service should carry out.

A node location service is a database of nodes position information. The system implements two main operations: position update to submit a node's current position, and position lookup to retrieve it. In Figure 1(a) node $N$ updates its current position $(x, y)$ submitting it to the service along with its unique identifier. Node $M$ in turn, queries the service looking for the position of node $N$, and receives back the correct answer.

On a Metropolitan Ad Hoc network, the absence of an infrastructure implies that nodes have to implement the location service themselves. First of all, the position database has to be distributed. Many distribuition schemes have been already proposed [1]. Essentially all or some of the nodes maintains position information, these are the location servers, and each of them maintains a copy of the whole or only a part of the database. A fair database distribution scheme involves all the nodes as potential location servers, making each responsible only 


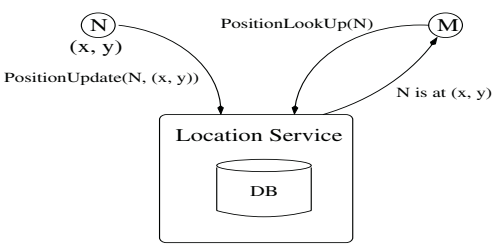

(a) Classic architecture

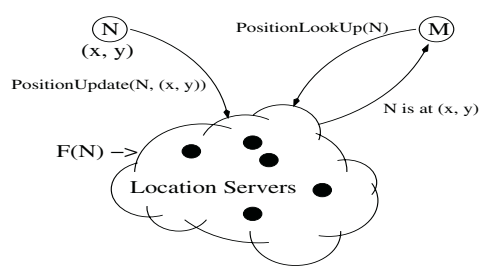

(b) Ad Hoc variation

Fig. 1. Location services.

for a part of the database. The location servers of a given node $N$ are identified through a function $F$ on the unique identifier (see Figure 1(b).

In the Virtual Home Region 2], a consistent hash function $H()$ maps each node unique identifier to the centre of a circular area called the home region. Those nodes located inside the home region of a given node $N$ are its potential location servers. When $N$ updates its position, it geographically forwards a request packet to its home region, where some nodes will eventually cache it. A position lookup proceeds similarly: when node $M$ needs to retrieve $N$ 's current position, it geographically forwards a position lookup request packet to $N$ 's home region, identified through the same hash function $H()$. Eventually, one of the location servers of $N$ will answer to the request. The hash function uniformly maps node identifiers over the network area, independently from the geographic nodes distribuition.

For the same node $N$, location servers in the Grid Location Service [3] are spread all over the network area. The density of location servers decreases logarithmically moving away from $N$. On one hand, position lookups are guaranteed to find closer location server. On the other hand, position updates have higher costs as they cover the whole network area. The system virtually partitions the network area in squares of increasing size, and tries to guarantee for $N$ the existence of a location server in each square. Inside a square, the location server is again identified through the node identifier, as the node that has the least greater identifier compared with $N$.

This paper presents a location service where the association between nodes and location servers is context aware. On a metropolitan area, nodes tend to assemble around well defined contexts, instead of uniformly distribute. As detailed in Section 2, urban user mobility patterns can be analysed and exploited to improve the location service perfomance together with its adaptability to the Ad Hoc dynamics. Section 3 briefly outlines the conclusions.

\section{Exploiting Metropolitan User Mobility Patterns}

On a metropolitan area, people do not uniformly distribute, but tend to assemble around hot spots. These are for example offices, shopping malls, theathers or similar places where people is plunged in a well defined context. Beside that, a 
user tends to frequent the same contexts, where often the same people can be met. Of daily habits change, but over long periods of time.

If thousends of PDA-equipped users are connected on a metropolitan Ad Hoc network, the observations above will result in users possibly aware of places where connectivity and node density are usually good. People can understand and easily remember hot spots locations. Moreover, this process could be directly supported using mobile hosts. For example each mobile host could maintain a ranking of positions where its user passed over and where connectivity and node density was good. Once a user identifies a hot spot context, the system can exploit it to colocate its location servers. The user will then compose its address with the unique identifier and the hot spot's coordinates $\left(X_{h s}, Y_{h s}\right)$, resulting in $U I D @\left(X_{h s}, Y_{h s}\right)$. This address format is not far from present email addresses like my_id@my_domain, where my_id identifies a mailbox in the domain my_domain. Finally, users distribute context aware addresses as present e-mail addresses are exchanged today. This give seekers nodes a way to find the location servers of sought-after nodes.

\subsection{Assumptions and Definitions}

Each node is able to sense its current position through an on-board GPS device. A standard GPS device samples the current position one time per second, $f_{G P S}=1$. Besides, routing is assumed to implement a geographic forwarding procedure, like those described in [1].

Consider a node $N$ and a hot spot with coordinates $X_{h s}, Y_{h s}$ specifyed by its user. The system potentially identifies the location servers of $N$, as those nodes currently located around the hot spot. More formally, the location servers of $N$ belong to its Friends set $F_{N}$, defined as the set of nodes located at distance less than or equal to a value $r$ from the point $X_{h s}, Y_{h s}$. Note that not all the $F_{N}$ nodes are location servers for $N$. Some of them might be unreachable, while others might have just entered the area, without having yet received a position update. Infact, node $N$ has no control over its $F_{N}$ area, and no knowledge about its location servers. The node periodically sends a position update request towards the $F_{N}$ area, simply asking to cache the position information for a limited amount of time. Infact, the context aware location service is a soft state system. Positions are only temporarily cached on location servers. This eliminates the problem of handling stale entries.

In order to be locatable, node $N$ must also deliver its $F_{N}$ references to its peers. As outlined before, the address of $N$ is composed by the unique identifier and the hot spot coordinates, $A d d r_{N}=\left[U I D_{N},\left(X_{h s}, Y_{h s}\right)\right]$. The hot spot coordinates are the centre of $F_{N}$, so that far away peers holding the address of $N$, will eventually retrieve its current position querying nodes towards this point. Consider at first the distance parameter $r$ as a constant value. Later on it is shown how this can vary dynamically according to the density of nodes in the area. 


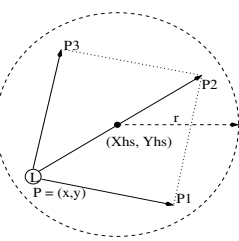

(a) Flooding

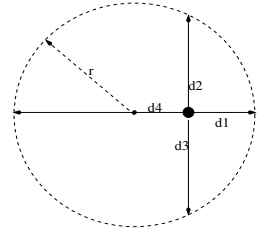

(b) Leaving

Fig. 2. Handling dynamics inside $F$.

\section{$2.2 \quad$ Position Update}

Node $N$ periodically updates its current position to its location servers, as the system is soft state. The caching time, and respectively the update frequency, vary accordingly to the node's mobility pattern (see Section [2.3). A postion update consists in the following steps:

1. An update request packet is geographically forwarded towards $F_{N}$. By definition, the forwarding procedure will deliver the packet to the reachable node $L$ that closest to the $F_{N}$ coordinates.

2. If $L$ is at distance greater than $r$, then a failure will return back to $N$. Otherwise, $L$ floods the position update request bounding to the $F_{N}$ area. Each node receiving the position update request caches $N$ 's position and broadcasts again the packet only if it belongs to $F_{N}$, otherwise it simply drops it.

3. Node $L$ collects acknoledgements from caching nodes and sends back a cumulative packet to $N$.

Nodes distribution inside $F_{N}$ can be far from uniform. In this case, the packet flooding described above, does not guarantee $N$ to have its position cached in a satisfying number of nodes spanning the $F_{N}$ area. To minimize this condition, node $L$ could drive the flooding to start also from other three points located inside $F_{N}$ as shown in Figure 2(a). Having the position $(x, y)$ of node $L$ and the $F_{N}$ coordinates $\left(X_{h s}, Y_{h s}\right)$, points $P_{1}, P_{2}$ and $P_{3}$ are simple orthogonal projections. The coordinates for the points are $P_{1}=\left(X_{h s}-y+Y_{h s}, Y_{h s}+x-X_{h s}\right), P_{2}=$ $\left(2 X_{h s}-x, 2 Y_{h s}-y\right), P_{3}=\left(X_{h s}+y-Y_{h s}, Y_{h s}-x+X_{h s}\right)$.

\subsection{Position Update Frequency}

Each node updates its current position periodically, with a frequency depending from its linear speed (or displacement). The faster the node moves linearly, the more frequent it updates its position.

Each node is assumed to move not faster than a maximum speed $S_{M A X}$. Moreover, a motionless node updates at a given base frequency $f_{b}$ to refresh its location servers. Given that, the position update frequency should range between $\left[f_{b}, K * f_{b}\right]$. The upper bound is $K$ times the base frequency, and has to be reached when the node moves at the maximun linear speed $S_{M A X}$. If $S_{n}$ 
indicates the current linear speed, given by the distance between the position at step $n$ and the position ar step $n$-1, then, assuming $f_{0}=f_{b}$ and $S_{0}=0$, the formula

$$
f_{n}=f_{n-1}+\frac{S_{n}-S_{n-1}}{S_{M A X}} *(K-1) * f_{b}=f_{b} *\left(1+(K-1) * \frac{S_{n}}{S_{M A X}}\right)
$$

has the right features, as $S_{n}$ ranges in $\left[0, S_{M A X}\right]$. Finally, considering $f_{G P S}$ and the cost of a position update operation, fixing $K * f_{b}=\frac{f_{G P S}}{H}$, where $H=10^{h}$ and $h$ is a natural number, results in having $f_{n} \in\left[\frac{f_{G P S}}{K * H}, \frac{f_{G P S}}{H}\right]$.

\subsection{Position Lookup}

A node $M$ willing to communicate with node $N$ has to have its address $A d d r_{N}=$ $\left[U I D_{N},\left(X_{h s}, Y_{h s}\right)\right]$. There are two cases:

1. $N$ is sufficiently close to $M$, so that they directly see each other. In this case there is no need to query the location service;

2. $N$ is far away from $M$, the two nodes do not directly see each other, and $M$ needs to query the location service to obtain $N$ 's position and furtherly use geographic forwarding to exchange packets with it.

A position lookup is needed in the second case. Node $M$ geographically forwards a position lookup request packet towards $\left(X_{h s}, Y_{h s}\right)$. Eventually a node selected by the forwarding procedure, caching $N$ 's position, will send back a lookup answer packet. If none exists, an error is reported to $M$. It is worth of note, that in order to lookup the current position of a node, the size $r$ of the $F_{N}$ area is not needed.

\subsection{Varying the Size of the Friends Set}

For a node $N$, the $F_{N}$ area has been defined with a fixed distance parameter $r$. A direct improvement is to let $r$ be variable in order to guarantee a percentage of successfull position lookups coming between subsequent position updates. Infact, it is possible that in the time frame between two position updates, enough caching nodes leave the $F_{N}$, determining the failure of incoming position lookups. The goal is to minimize the probability of this event.

As described in Section 2.2, the leader $L$ of the position update request flooding, returns a cumulative acknowledgement to node $N$. This contains at least the number of caching nodes in $F_{N}$. Let this number be $n$. As shown in Figure 2(b) consider one of these nodes moving out of $F_{N}$ at a given discrete speed $s \in\left[1, \ldots, S_{M A X}\right]$, choosing one of the directions $\overrightarrow{d_{1}}, \overrightarrow{d_{2}}, \overrightarrow{d_{3}}, \overrightarrow{d_{4}}$.

Considering only these four directions is of course an approximation. Suppose, without loss of generality, to have the node positioned along the radius at 
point $\left(x, Y_{h s}\right)$ with $x$ variable, and the position update frequency to be $f$. Approximating with a uniform nodes distribution inside $F_{N}$, the probability $P\left\{\right.$ out $\left._{f}\right\}$ that the node leaves the area before the next position update, is given by:

$$
\int_{0}^{r} \sum_{s=1}^{S_{M A X}} \sum_{i=1}^{4} P\left\{\text { out }_{f} \mid S=s, D=\vec{d}_{i}, x=d x\right\} P(S=s) P\left(D=\overrightarrow{d_{i}}\right) P(x=d x) d x
$$

where $P\left\{\right.$ out $\left._{f} \mid S=s, D=\overrightarrow{d_{i}}, x=d x\right\}$ is 0 or 1 depending on the frquency $f$, the position $X$, the speed $s$ and the direction $\vec{d}$. The condition $P\left\{\text { out }_{f}\right\}^{k} \leq 1 \%$ gives the minimum number of nodes $k$ that the $F_{N}$ should contain to guarantee the success of incoming position lookups. By comparing $k$ with the returned number of caching nodes $n$, a policy to vary the size of $F_{N}$ can be implemented. The policy could be continuos, as $P\left\{\right.$ out $\left._{f}\right\}$ depends from $r$, or discrete, for example advancing at multiples of $T_{x}$, equal to the MAC layer maximum transmission range. This is currently under evaluation.

\section{Conclusions and Future Work}

This short paper describes the key polices of a context aware location service. The role of a location service is essential at the networking layer when the size of an Ad Hoc network grows up to a metropolitan scale, but nevertheless it could also be used by location aware middlewares and applications. None of the previously proposed location services are context aware. This system uses context to realize policies maximizing the percentage of successful operations, while keeping costs comparable to other approaches like VHR and GLS. The results of a preliminary performance evaluation could be found in [4].

\section{References}

1. M. Mauve, J. Widmer, and H. Hartenstein. A Survey on Position-Based Routing in Mobile Ad-Hoc Networks. IEEE Network, Vol. 15 (2001), Nr. 6, pg. 30-39, 2001.

2. S. Giordano, and M. Hamdi. Mobility Management: The virtual home region. Technical report, EPFL-(ICA), Lausanne, Switzerland, October 1999.

3. J. Li, J. Jannotti, D. S. J. De Couto, D. R. Karger, and R. Morris. A scalable location service for geographic ad hoc routing. Proc. of the 6th Annual ACM/IEEE Int. Conf. on Mobile Computing and Networking (MOBICOM) 2000, pg. 120-130, Boston, MA, USA, 2000.

4. M. Conti, E. Gregori, and G. Turi. Desing and Analysis of a Context Aware Node Location Service for Ad Hoc Networks. Submitted for publication, 2003. 